\title{
Plastic deformation of nanocrystalline nickel
}

\author{
WU XiaoLei \\ State Key Laboratory of Nonlinear Mechanics, Institute of Mechanics, Chinese Academy of Sciences, Beijing 100190, China \\ (email: xlwu@imech.ac.cn)
}

\begin{abstract}
A high-resolution electron microscopy study has uncovered the plastic behavior of accommodating large strains in nanocrystalline (NC) Ni subject to cold rolling at liquid nitrogen temperature. The activation of grain-boundary-mediated-plasticity is evidenced in $\mathrm{NC}-\mathrm{Ni}$, including twinning and formation of stacking fault via partial dislocation slips from the grain boundary. The formation and storage of $60^{\circ}$ full dislocations are observed inside NC-grains. The grain/twin boundaries act as the barriers of dislocation slips, leading to dislocation pile-up, severe lattice distortion, and formation of sub-grain boundary. The vicinity of grain/twin boundary is where defects preferentially accumulate and likely the favorable place for onset of plastic deformation. The present results indicate the heterogeneous and multiple natures of accommodating plastic strains in NC-grains.
\end{abstract}

nanocrystalline metal, plastic deformation, deformation twinning, dislocation, microstructure

The molecular dynamics (MD) simulations have predicted that the nanocrystalline (NC) metals deform via the grain-boundary-mediated-plasticity(GBMP ${ }^{[1-4]}$. The grain boundaries (GBs) are sources and sinks of dislocations, and the dislocations are emitted in the form of partial dislocations, one at a time. The generalized planar fault energy curves play a critical role in the activation of partial dislocations from $\mathrm{GBs}^{[3]}$. The slips of partial dislocations occur in three modes depending on the applied stress $\frac{[1-3]}{}$, including: (1) the emission of the leading partial (Burgers vector $\boldsymbol{b}_{1}$ ) from GB, leading to the formation of stacking faults; (2) successive emission of leading and trailing partial $\left(\boldsymbol{b}_{2}\right)$ on the same slip plane with the trailing partial catching up with the leading, resulting in a perfect dislocation $\left(\boldsymbol{b}=\boldsymbol{b}_{1}+\boldsymbol{b}_{2}\right)$ which is then absorbed by GB; (3) emission of twin partials on adjacent slip planes from the GB, causing deformation twins. MD simulations have predicted that $\mathrm{NC}-\mathrm{Al}^{[1,2]}$ prefers to deform by extended partials and twinning whereas $\mathrm{NC}-\mathrm{Ni}{ }^{[3]}$ deforms only by extended partials.

Deformation twinning has recently been identified as a contributing plastic deformation mechanism in $\mathrm{NC}-\mathrm{Al}^{[5,6]}, \mathrm{Cu}^{[7]}, \mathrm{Pd}^{[8]}$ and $\mathrm{Ta}^{[9]}$ as evidenced by high- resolution electron microscopy (HREM) observations. The NC-Al case is especially interesting, as the facecentered-cubic metals of high stacking fault energy have little or no chance of undergoing deformation twinning in their coarse-grained form. So far, most experimental evidences for GBMP have been obtained in NC-metals that were subjected to complicated stress states and high stress levels, such as in indentation, high-pressure torsion, high-rate cold rolling, and ball milling. Recently, the GBMP, including twinning and stacking fault formation, has been observed in $\mathrm{NC}-\mathrm{Ni}$, for the first time under uniaxial tensile test conditions ${ }^{[10]}$. This indicates that the GBMP controls the mechanical property, e.g., strength and plasticity of NC-metals. Kumar et al. ${ }^{[11]}$ observed deformation twins and dislocations on an NC-Ni foil in situ deformed inside a TEM. However, in such a TEM foil most of the deformation is associated with the advancement of a crack tip with complex stress state. Meanwhile, when only a few grains are sitting on

Received March 22, 2009; accepted April 21, 2009

doi: 10.1007/s11431-009-0224-z

${ }^{\dagger}$ Corresponding author (email: xlwu@imech.ac.cn)

Supported by the National Nature Science Foundation of China (Grant Nos. 50571000, 10721202) and the National Basic Research Program of China ("973" Program) (Grant No. 2004CB619305) 
top of each other near the free surfaces, dislocation activities, diffusional processes, and changes in the GB structures may have been enhanced. As such, it is questionable if in situ observations in thin TEM foils are relevant to bulk deformation. Budrovic et al. ${ }^{[12]}$ conducted an in situ X-ray diffraction studies of NC-Ni subjected to tensile deformation and showed no irreversible peak broadening, indicative of the formation of extended faults during tension that is in line with MD simulations ${ }^{[3]}$. They considered that the trailing partial erases the stacking fault as it runs across the grain to reach the opposite GB, leaving no footprint for peak broadening. However, if the GB emission of the leading partial is favored (e.g., the shear stress is parallel to its Burgers vector $\boldsymbol{b}_{1}$ ), it would be much more difficult to emit the trailing partial having an unfavorable orientation. Statistically, it is not likely that all the stacking faults left by leading partials would get erased by trailing partials.

Dislocations play a dominant role also in GBMP of NC-metals. Based on MD simulations ${ }^{[3]}$, the dislocations active in deformation are all absorbed by GBs with no dislocation debris left. However, dislocations were detected by in situ and ex situ TEM examinations of plastically deformed $\mathrm{NC}-\mathrm{Ni}{ }^{[11]}$ and $\mathrm{Cu}^{[13]}$ respectively. Rapid changes in TEM diffraction contrast have been interpreted as dislocation movements upon straining. In NC-grains, the conventional two-beam and weak-beam TEM imaging conditions are difficult to operate for characterizing the nature of dislocations. So far there has been no reported success in using these techniques for quantitative identification of individual dislocations. Indeed, a convincing TEM identification of dislocations in NC-grains is a non-trivial task.

There exist evident differences on partial dislocation processes in NC-metals between MD simulations ${ }^{[3]}$ and experimental results ${ }^{[5-11]}$ or even among MD simulations ${ }^{[1-3]}$. This is mainly due to the ultra-high strain rate $\left(10^{7}-10^{8} \mathrm{~s}^{-1}\right)$ and resultant large stresses involved in MD simulations as compared with experimental tests, together with the differences in material features, e.g., grain size, size distribution, etc. ${ }^{[14,15]}$.

The deformation physics of NC-metals, such as twinning and formation of extended faults and dislocations, closely relates to their mechanical property. $\mathrm{Lu}$ et al. ${ }^{[16,17]}$ observed the formation and storage of high density dislocations in nano-twinned $\mathrm{Cu}$, leading to work hardening capacity and high ductility. Both deformation twinning with inverse grain size effect and twinning without producing macroscopic strains in NC-metals will play a significant role in the strength and ductility of NC-metals $\frac{[18,19]}{}$.

The partial dislocation slips related to GBMP, e.g., deformation twinning and dislocation activity, are thermally activated $\frac{[14,15,20]}{}$. Especially, NC-metals show a stronger-than-normal temperature and strain rate dependence of the flow stresses due to the fact that the relaxation/adjustment in the grains and GBs is depressed, and the activation of dislocations would then rely largely on the local stress intensity (depending on the applied stresses, internal stresses, and the stress field of the leading partial $)^{[14,15]}$. Cold rolling at liquid nitrogen temperature (LNT) was used because in a tensile test the $\mathrm{NC}-\mathrm{Ni}$ fails at a small strain of only a few percent, making it impossible to observe how the NC-grains evolve at large plastic strains, which would otherwise annihilate and undergo de-pinning much faster at room temperature. Hence, the present study aims at observing how NC-grains respond to the imposed deformation by subjecting NC-Ni to large cold strains of 0.2 at LNT, to facilitate the observation of deformation patterns and defect accumulation.

\section{Experimental}

Fully dense NC-Ni sheets were procured from Goodfellow Inc. The as-received foil was $150 \mu \mathrm{m}$ thick and of $99.9 \%$ purity. The NC-Ni samples of $4 \mathrm{~mm} \times 16 \mathrm{~mm}$ in size were cold-rolled at LNT to obtain a rolling strain of 0.2 . The rolling rate was approximately $10^{-2} \mathrm{~s}^{-1}$. The microstructures were examined using a HREM, JEM2010F operated at $200 \mathrm{kV}$. The TEM specimens were prepared by conventional twin-jet polishing technique. The as-received NC-Ni foil was examined before rolling. After examining about 100 grains, we concluded that the presence of growth twins and dislocations was relatively low ${ }^{[10]}$. And, no grain growth was observed after LNT rolling.

\section{Results and discussion}

Figure 1 is the HREM image showing deformation twins in an NC-grain. Three twin plates are visible. These twins heterogeneously nucleate at the GB and grow into the grain interior via the emission of partial dislocations 


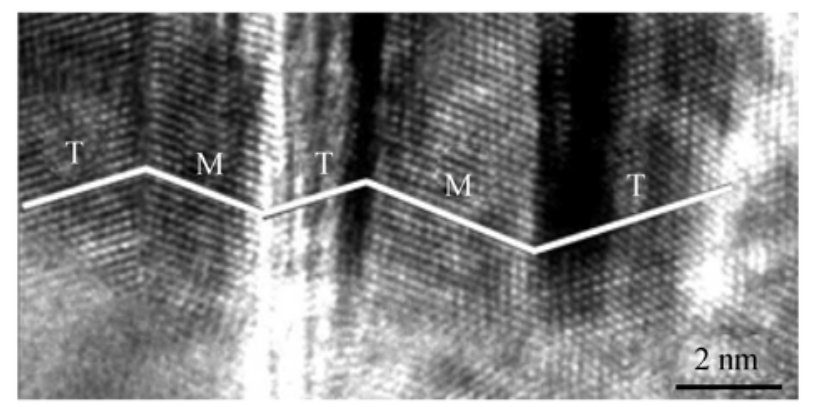

Figure 1 HREM lattice image showing deformation twins in NC-Ni (T: twins; M: matrix).

from GB, consistent with the prediction of MD simulations ${ }^{[1]}$. Extensive observations reveal that deformation twinning dominates in plastic deformation during LNT rolling of NC-Ni. The higher strength at LNT rolling produces larger difference in the resolved shear stress between the leading and trailing partials with different Schmid factors, which will reduce the probability of the trailing partial and favor twin nucleation that requires partials with the same Burgers vector as for the leading partial. Thus, twinning may become kinetically competitive at LNT rolling as compared with tension deformation ${ }^{[10]}$.

The formation of dislocations is also a main plastic response during LNT rolling of NC-Ni. Figure 2 shows one dislocation marked by a " $\mathrm{T}$ " and Burgers circuit enclosing the dislocation core. As the electron beam and the dislocation line are parallel to [011], the Burgers vector of the dislocation is determined to be $1 / 2[101]$ or $1 / 2[1 \overline{1} 0]$, which has an angle of $60^{\circ}$ (or $120^{\circ}$ ) with

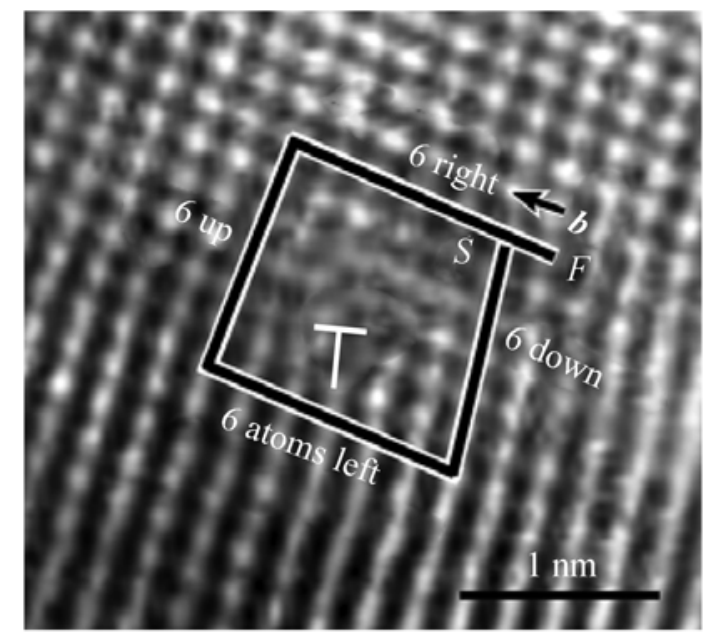

Figure 2 HREM lattice image showing full dislocation of $60^{\circ}$ type. A Burgers circuit starting at $S$ and ending at $F$ was drawn to enclose the dislocation core marked by a "T". The electron beam and the dislocation line are parallel to [110], and the Burgers vector $\boldsymbol{b}=1 / 2$ [011] or $1 / 2$ [101]. respect to the dislocation line. For this reason, the dislocation is referred to as a $60^{\circ}$ one. The dislocations of $60^{\circ}$ type are glissible along (111) planes. The dislocations form mainly in the form of $60^{\circ}$ type during plastic deformation of NC-Ni.

Apart from the individual dislocation, the dislocation dipole, i.e. dislocations with opposite signs of Burgers vector, is often seen to reside near the GB. One example is shown in Figure 3. Three dislocations as indicated by "Ts" are all $60^{\circ}$ full dislocations by Burgers circuit shown in the inset.

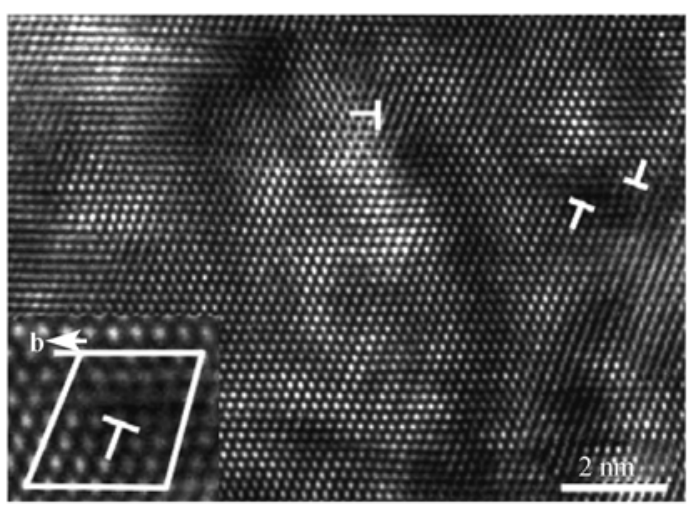

Figure 3 HREM lattice image showing individual dislocations and dislocation dipoles. The inset is a Burgers circuit enclosing the dislocation core marked by a " $\mathrm{T}$ ".

Figure 4 is a HREM image showing the partial dislocation slips in various ways just in one NC-grain. Deformation twinning and the formation of dislocation and extended faults occur simultaneously. The micro-twin of only two atomic-spacing thick is visible as indicated by a triangle. It is formed by absorbing extended partials from neighboring planes, i.e., via dynamic overlapping of stacking faults on adjacent planes ${ }^{[1]}$. Such a mechanism occurs when the concentration of the extended dislocations inside the grain is rather high ${ }^{[14]}$. The twin boundary (TBs) often acts as the barrier for dislocation slip, leading to pile-up and accumulation of dislocations near the TB and causing lattice distortion and curvature, as shown in Figure 5.

The dislocations are observed to form and reside near the GB, as shown in Figure 6. Two pairs of lines indicate the evident change of crystallographic orientations from the GB into the grain interior. Large plastic strains can be accommodated for the most part by dislocation activities and storage. We found that the vicinity of the GBs, and TBs as well, is where defects preferentially 


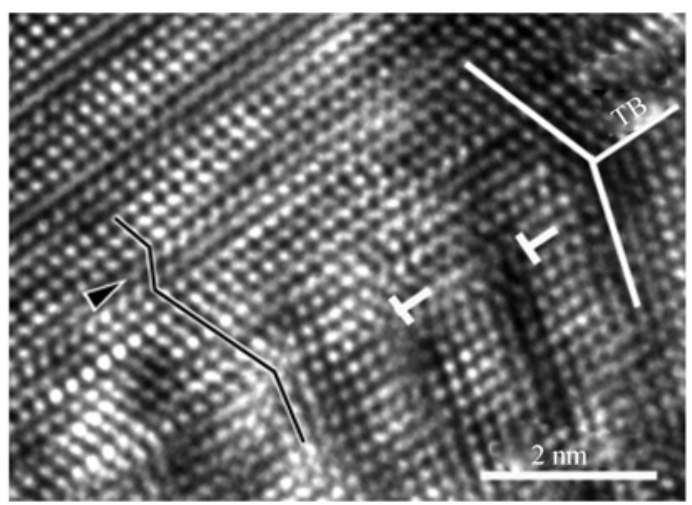

Figure 4 HREM lattice image showing deformation twin, dislocations marked by "Ts" and micro-twin indicated by a triangle.

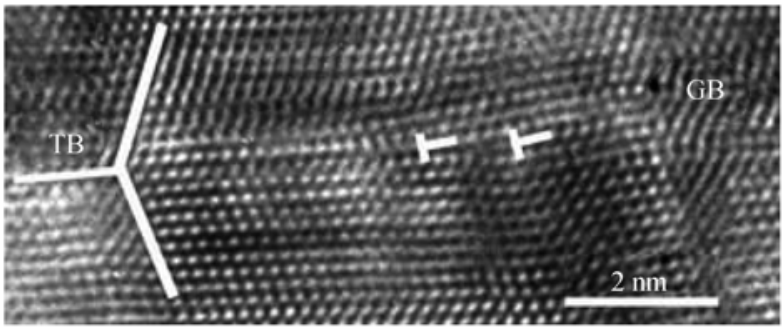

Figure 5 HREM lattice image showing deformation twins and dislocations ("Ts").

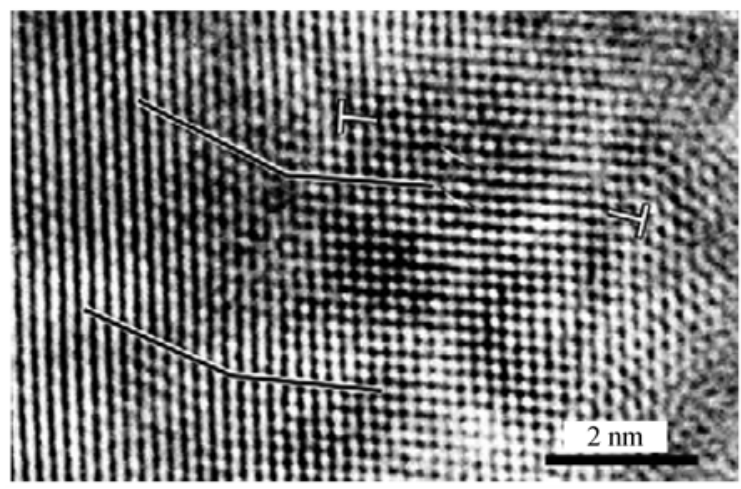

Figure 6 HREM lattice image showing lattice distortion and dislocations ("Ts") near the grain boundary.

accumulate and likely the favorable place for plastic deformation in NC-grains.

Figure 7 is a HREM micrograph obtained from the [011] zone axis. Severe lattice distortion occurs, as indicated by the formation of the square lattice labeled $\mathrm{B}$ in contrast with the parallelogram lattice labeled A with the [011] zone axis. This is due to several dislocations (indicated by "Ts") to stick up at the boundary between the square lattice and parallelogram lattice, resulting in gradually tilting of $(\overline{1} \overline{1} 1)$ planes. Such a change of the crystallographic orientation of the $(\overline{1} \overline{1} 1)$ planes even-

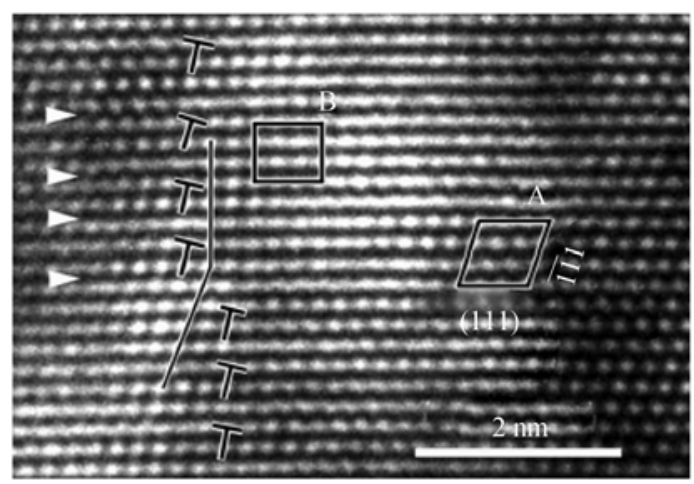

Figure 7 HREM lattice image showing severe lattice distortion. Boxed areas labeled A and B indicate parallelogram and square lattice, respectively. Dislocations and SFs are indicated by "Ts" and triangles, respectively.

tually leads to the formation of square lattice. In addition, there are stacking faults, indicated by the arrows.

Figure $8(\mathrm{a})$ is a low-magnification image of a $\sim 50 \mathrm{~nm}$ grain, showing several areas with clear strain contrast due to localized lattice distortion of dislocation cores. Interestingly, these dislocations form a dislocation sub-boundary as indicated by the arrow. The details are given in the HREM image of Figure 8(b). This dislocation array, in fact, leads to the formation of a low-angle boundary of $\sim 4^{\circ}$ misorientation of (111) planes at the two sides of the dislocation array. Hence, this dislocation sub-boundary leads to grain refinement in tiny NC-grains.

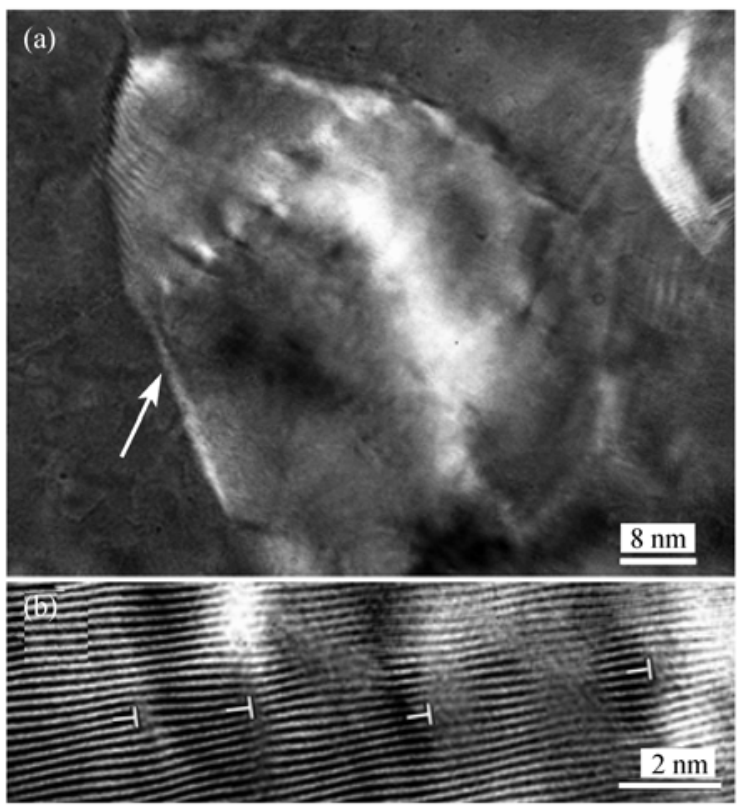

Figure 8 (a) TEM micrograph of an NC-Ni grain after LNT rolling. The arrow indicates an array of contrast due to the strains associated with dislocation cores; (b) HREM image of the dislocations ("Ts") that form a low-angle boundary of $\sim 4^{\circ}$ misorientation. 
Deformation twinning (Figures 1, 4 and 5) and formation of extended faults (Figures 4 and 7) and dislocations(Figures 2-7) are unequivocally identified in LNT rolled NC-Ni. This indicates the activation of GBMP during LNT rolling NC-Ni, similar to that of LNT tensile tests of $\mathrm{NC}-\mathrm{Ni} i^{[10]}$.

We have directly imaged and identified full dislocations of $60^{\circ}$ type with individual and dipole patterns. The full dislocations not only all traverse the grains during deformation leaving no debris behind but also are capable of accumulating inside NC-grains. The dislocation configurations observed, including the dislocation piles near $\mathrm{TB} / \mathrm{GB}$, suggest that the interactions with other defects, in particular TB/GB may be the origin of stabilizing forces that trap dislocations inside NC-grains. In addition, the interaction (pinning) forces at LNT are apparently sufficient to keep some of the dislocations from propagating and disappearing into the opposing GBs. Discounting those right inside the GBs, the dislocation density inside the NC-grains was estimated to be in the range of $5.2 \times 10^{12}-2.8 \times 10^{15} \mathrm{~m}^{-2}$. In tiny $\mathrm{NC}$ grains, the population of Frank-Read sources is expected to be low and their operation would require large stresses due to the high curvatures. Our postmortem HREM observations revealed the formation of full dislocation, possibly emanating from both $\mathrm{GB}$ and $\mathrm{TB}$ as suggested by MD simulations. Note also that for $\mathrm{NC}-\mathrm{Ni}$, MD simulations were not able to reveal dislocation processes after the emission of a leading partial dislocation from GBs, due to the very limited simulation time ${ }^{[3]}$.

Ashby ${ }^{[21]}$ and Kocks ${ }^{[22]}$ proposed a composite model of plastic deformation: the deformation of each grain may be separated into a uniform deformation region in the grain interior and a local, nonuniform deformation zone in the GB regions. A microscopic incompatibility region may exist in the vicinity of $\mathrm{GB}$, forming a GB region containing high densities of dislocations. The GBs may act as dislocation sources and sinks, and

1 Yamakov V, Wolf D, Phillpot S R, et al. Dislocation processes in the deformation of nanocrystalline materials. Nat Mater, 2002, 1(1): $45-48[$ doi]

2 Yamakov V, Wolf D, Phillpot S R, et al. Deformation-mechanism map for nanocrystalline metals by molecular-dynamics simulation. Nat Mater, 2004, 3(1): 43-47[doi]

3 van Swygenhoven H, Derlet P M, FrÃseth A G. Stacking fault energies complex deformation structures, i.e. patterns of accumulated dislocations induced by plastic deformation, have been frequently observed in the GB region. The GB region can, therefore, be more strained than the grain matrix and somewhat stronger than the matrix. Hansen ${ }^{[23]}$ exhibited unique deformation patterns in the vicinity of GB. Different assumptions and predictions can be found in the literature for plastic deformation in NC-metals. Some models do use the concept of a GB zone, deforming differently from the grain interior, but not composed of dislocation structures ${ }^{[24]}$. In our case, however, the GB and its vicinity are the place that plastically deforms first and the most, as shown in Figures 5 and 6. Especially, it is at GB that cooperating processes such as dislocation emission, dislocation absorption, shearing, atomic shuffling and GB diffusion take place. All these operative processes, including the dislocation activities, allow the required deformation compatibility to be satisfied at the GB. Furthermore, various types of defects are observable as shown in Figures 4, 5 and 7. There appear to be multiple different deformation responses for accommodating plastic strain even in one single NC-grain.

\section{Conclusions}

(1) The grain-boundary-mediated-plasticity is evidenced during LNT cold rolling of NC-Ni, including deformation twinning and formation of extended faults and dislocations.

(2) The full dislocations of $60^{\circ}$ type may form and store at or near the grain boundary and twin boundary in NC-grains. The dislocations may pile-up, leading to severe lattice distortion and formation of low angle boundary.

(3) The inhomogeneous defect accumulation prefers to occur in the vicinity of the GB. The strain accommodation has multiple pathways and is inhomogeneous from place to place in the NC-grain.

and slip in nanocrystalline metals. Nat Mater, 2004, 3(6): $399-403$ [doi]

4 Schiøtz J, Jacobsen K W. A maximum in the strength of nanocrystalline copper. Science, 2003, 301(5638): 1357-1359[doi]

5 Chen $\mathrm{M} \mathrm{W}$, Ma E, Hemker $\mathrm{K} \mathrm{J}$, et al. Deformation twinning in nanocrystalline Aluminum. Science, 2003, 300(5623): $1275-1277[$ doi] 
6 Liao X Z, Zhou F, Lavernia E J, et al. Deformation twins in nanocrystalline A1. Appl Phys Lett, 2003, 83(24): 5062-5064[doi]

7 Liao X Z, Zhao Y H, Srinivasan S G, et al. Deformation twinning in nanocrystalline copper at room temperature and low strain rate. Appl Phys Lett, 2004, 84(4): 592-594[doi]

8 Rösner H, Markmann J, Weissmüller J. Deformation twinning in nanocrystalline Pd. Phil Mag Lett, 2004, 84(5): 321 - 334[doi]

9 Wang Y M, Hodge A M, Biener J, et al. Deformation twinning during nanoindentation of nanocrystalline Ta. Appl Phys Lett, 2005, 86(10): 101915 [doi]

10 Wu X L, Zhu Y T, Chen M W, et al. Twinning and stacking fault formation during tensile deformation of nanocrystalline Ni. Scr Mater, 2006, 54(9): 1685-1690[doi]

11 Kumar K S, Suresh S, Chisholm M F, et al. Deformation of electrodeposited nanocrystalline nickel. Acta Mater, 2003, 51(2): $387-405$ [doi]

12 Budrovic $Z$, van Swygenhoven H, Derlet P M, et al. Plastic deformation with reversible peak broadening in nanocrystalline nickel. Science, 2004, 304(5668): 273-276[doi]

13 Youssef K M, Scattergood R O, Murty K M, et al. Ultrahigh strength and high ductility of bulk nanocrystalline copper. Appl Phys Lett, 2005, 87(9): 091904[doi]

14 Wu X L, Zhu Y T, Ma E. Predictions for partial- dislocation-mediated-processes in nanocrystalline $\mathrm{Ni}$ by generalized planar fault energy curves: An experimental evaluation. Appl Phys Lett, 2006,
88(12): 121905 [doi]

15 Wu X L, Qi Y, Zhu Y T. Partial-mediated slips in nanocrystalline Ni at high strain rate. Appl Phys Lett, 2007, 90(22): 221911[doi]

16 Lu L, Shen Y F, Chen X H, et al. Ultrahigh strength and high electrical conductivity in copper. Science, 2004, 304(5669): 422 - 426[doi]

17 Lu L, Chen X, Huang X, et al. Revealing the maximum strength in nanotwinned copper. Science, 2009, 323(5914): 607-610[doi]

$18 \mathrm{Wu}$ X L, Zhu Y T. Inverse grain-size effect on twinning in nanocrystalline Ni. Phys Rev Lett, 2008, 101(2): 025503[doi]

19 Wu X L, Liao X Z, Srinivasan S G, et al. New deformation twinning mechanism generates zero macroscopic strain in nanocrystalline metals. Phys Rev Lett, 2008, 100(9): 095701[doi]

20 Wang Y M, Ma E. On the origin of ultrahigh cryogenic strength of nanocrystalline metals. Appl Phys Lett, 2004, 85(14): $2750-2752[$ doi]

21 Ashby M F. The deformation of plastically non-homogeneous materials. Philos Mag, 1970, 21(170): 399-424[doi]

22 Kocks U F. The relation between polycrystal deformation and single crystal deformation. Metall Mater Trans B, 1970, 1(5): 1121-1143

23 Hansen N. Polycrystalline strengthening. Metall Mater Trans A, 1985, 16(12): 2167-2190[doi]

24 Benson D J, Fu H H, Meyers M A. On the effect of grain size on yield stress: Extension into nanocrystalline domain. Mater Sci Eng A, 2001, 319-321: 854-861[doi] 